\title{
Web-BASEd LeARning IN Periods OF CRISIS: REFLECTIONS ON THE IMPACT OF COVID-19
}

\author{
Stella Chiemeke ${ }^{1}$ and Omokhagbo Mike Imafidor ${ }^{2}$ \\ ${ }^{1}$ Department of Computer Science, University of Benin, Benin City, Nigeria \\ ${ }^{2}$ Africa PPP Advisory Nigeria Limited, Abuja, Nigeria
}

\begin{abstract}
Education systems and its actors are generally responding to quarantine and large-scale shutdown (partial) of cities with a sudden shift to Web-Based Learning. However, given that a pandemic of this nature and scale is novel, there is a knowledge gap as to how teachers and learners should respond to the shift, and what the likely impact and the key considerations should be. This study aims to extrapolate and theorize from the existing knowledgebase about the use of Web-Based Learning, as well as from an expert and practitioner wisdom and experience, to offer high-level guidance for policymakers and education system actors that are forced to make decisions in fast-moving and very challenging circumstances with little guidance or relevant experience. It is an early attempt at theorizing the impact of the pandemic on two key actors (Learners and Teachers) and one interface (Content), all across eight dimensions of learning. The analysis is based on Khan's (2001) dimension of Web-Based Learning and Anderson's (2011) Model of Online Learning. Overall, we posit based on experience and practice, that the pandemic has delivered severe shocks to both the demand and supply side of Web-Based Learning, with Leaners, Teachers, and Content all significantly affected. While we hypothesize a general drop in the quality of teaching and learning in the short run, we expect the opposite to be the case in the long run, when the demand and supply side self-correct, albeit guided by strong government and market institutions.
\end{abstract}

\section{KEYWORDS}

Web-Based Learning, COVID-19, Learners.

\section{INTRODUCTION}

\subsection{Background}

Learning dynamics have been significantly influenced on many levels by the swift expansion of the internet and increasing software capabilities. With COVID-19 in the mix, there is an ambiguity as to the impact it will have on Web-Based Learning. This is critical, as the crisis has delivered a combination of shocks as never experienced before; shocks to both demand, supply, market, and information.

The scale and severity of the impact of COVID-19 in well-resourced healthcare systems globally, has been beyond anything witnessed in recent history; talk less developing countries with poor health institutions. The way of life is rapidly changing and leaders and citizens across the globe are now being faced with the challenge of a lifetime. Interestingly, the ravaging epidemic has threatened not just the healthcare systems, but also other critical aspects of human life such as education. This is especially important, as education and health are both critical components of human capital development, with a significant theoretical and empirical basis for the assertion that they are both essential for economic growth and development (see Todaro and Smith, 2011; 
Romer, 2011). Indeed, human capital and particularly education have arguably been the most significant factor in regional differences in levels of development (Gennaioli, La Porta, Lopezde-silanes and Shleifer, 2011).

The education landscape has significantly evolved over the years, with web-based learning learning over the web- assuming a new dimension and revolutionizing education, given the enhancement it has made possible in education delivery and innovative pedagogic strategies. It has tackled time and place barriers to teaching, as it stretches traditional brick and mortar classrooms into learning spaces with computer and communication technologies to connect teachers and learners across time and space. A growing number of schools globally are now offering virtual education programs. Across the web, we also find a significant number of platforms offering virtual/online courses, and resources for customizing and delivering courses online. Some companies are utilizing the web to conduct training for their employees. Some terminologies used to explain different implementations of this internet-enabled environment for learning includes online learning, virtual classes, distance education, interactive learning, and cyber courses. While the growth of this web-based learning has been unprecedented in more advanced climes (despite few challenges as stated in The European-wide e-Learning Recognition Review Report, 2015), the developing world seems to be far behind, due to legacy challenges such as internet penetration, IT literacy, upfront technology investment cost, amongst several others (see Nagunwa \& Lwoga 2012; Sanga, Magesa, Chingonikaya, \& Kayunze, 2013; Dalton and Gren, 2015; Moeketsi, Matsephe \& Victor, 2018 ).

Given the increasing assuming role of web-based learning in education globally, various scholars have examined its efficiency, effectiveness, and adoption (see Ghavifekr \& Rosdy, 2015; Sudha, \& Amutha, 2015; Yongxia \& Peili 2018; Titiana, 2019). However, these studies have been conducted with an implicit but fundamental assumption of stable societies. COVID-19 has challenged this researches, as it has delivered severe and unimaginable shocks to every sphere of the society, creating the need for a new evidence base for the efficiency, effectiveness, and delivery of web-based learnings in periods of crisis. This is critical, as the lock-down of cities, and the shutdown of schools has triggered a rush in the utilization of this web-technology by various educational institutions; and if all stakeholders (from both the supply and the demand side, and regulators) are not properly guided, this rapid increase portends significant and farreaching implications, that if left in the dark, could trigger occurrences and or practices that have the potential of causing a deterioration in education outcomes, and thus development goals.

\subsection{Statement of Problem}

The spread of the Coronavirus disease (COVID-19) has triggered disruptions to normal life; countries have mandated temporary school closures, leaving a significant number of children and youth out of school. Extended school closures carry the potential to trigger not only short-term effects such as loss of learning, but also medium to long term human capital loss and weakened economic opportunities. To help mitigate the loss of learning, stakeholders in the education and teaching/training system (teachers and learners) are now pursuing options to utilize Web-Based Learning to manage and cope with the crisis.

Despite the above, very little research attention has been paid to documenting and analyzing the impact of the shock on education systems, as they move quickly and at scale to provide WebBased Learning when all or many schools are closed, and learners rapidly taking up learning online from their homes given restrictions to movement. 


\subsection{The objective of the Study}

This study aims to extrapolate and theorize from the existing knowledge base about the use of Web-Based Learning, as well as from an expert and practitioner wisdom and experience, to offer high-level guidance and 'rules of thumb' for policymakers and education system actors that are forced to make decisions in fast-moving and very challenging circumstances with little guidance or relevant experience.

Drawing from theory, this paper first reviews the conceptual framework for examining WebBased Learning and ultimately educational outcomes within the context of Web-Based Learning. We then proceed to examine the theoretical model for Web-Based Learning, with a focus on the key actors, interface, and interaction. Next, the study theorizes (based on experience and practice), the impact of COVID-19 on the key actors and interface in the Web-Based Learning model, across all the dimensions of teaching and learning, while making relevant recommendations. The analysis will cover how access to quality education is impacted in both the short run and in the long run.

\section{Conceptual Framework for EXamining Web-BASEd LeARNing}

There is no clear definition of Web-Based Learning (Titiana, 2019), and a review of the literature shows that it has been used interchangeably with terms such as "virtual learning", "technologybased learning", "network learning", "online learning", "multimedia-based learning", "Webenhanced learning", "Internet-enabled learning", "Web-based learning environment", and similar terms. A deep review of the literature suggests four key technological attributes, that when present, can be referred to as Web-Based Learning or any of its related terminologies. These attributes, according to Said (2010) are; (a) It is delivered online through the Web; (b) It communicates content that meets particular learning goals aligned with the curriculum; (c) It is designed based on a learning theory and pedagogical strategy; (d) It contains elements that are reusable

A starting point for evaluating web-based learning will be the examination of the environment for web-based learning. This paper builds on Khan's (2001) framework and presents an eightdimension framework that covers the full gamut of the environment of web-based learning in education. The dimensions are multi-disciplinary, with several theoretical and practical issues. These factors help to create a meaningful web-based learning environment, with many of them systemically interdependent and interrelated. A systemic and in-depth understanding of these factors will help in the full exploration of the impact of COVID-19 on web-based learning.

The eight dimensions of the web-based learning environment are; pedagogical, technological, interface design, evaluation, management, resource support, ethical, and institutional. Each dimension has several sub-dimensions, each consisting of items focused on a specific aspect of a Web-based learning (WBL) environment. The figure below shows the eight dimensions of the web-based learning environment. 


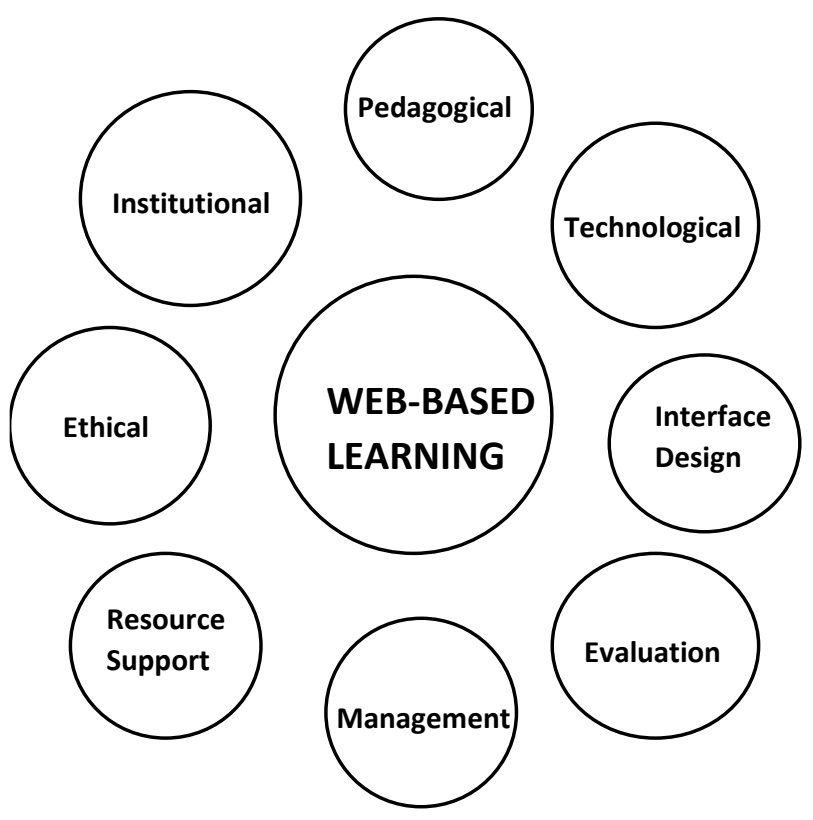

Fig. 1. Eight dimensions of Web-Based Learning Environment (Khan, 2001)

i. Pedagogy Dimension: Teaching and learning are the core of the pedagogical dimension of Web-based learning. This dimension focuses on matters relating to aims and objectives, approach to design, organization, delivery strategies and methods, and medium of Webbased learning environments. According to Kwanjai (2014), Web-Based Learning and Pedagogy can be considered as natural partners, where pedagogies are key determinants of the approaches and their effectiveness in the process of delivery and assessment of learning.

ii. Technological Dimension: The technological dimension considers issues relating to available technology infrastructure and its utilization as it relates to Web-based learning. The technological infrastructure for web-based learning includes technology planning, guidelines, software, computer hardware, and related technologies, operating system, Internet connectivity, and Internet services for instructors and learners.

iii. Interface Dimension: The interface design dimension refers to the appearance, navigability, and user-friendly nature of the web-based learning platform. It takes into cognizance, the overall appearance of Web-based teaching and learning programs. This dimension includes page and site design, content design, navigation, and usability testing.

iv. Evaluation Dimension: The evaluation dimension is a critical component of the web-based learning environment, as it allows for learning, customization, and adaptation. It ensures that the system is not abused, and the system is reliable. It encompasses an assessment of learners and evaluation of the overall instruction and learning environment, juxtaposed with the goal of the Web-Based Learning.

v. Management Dimension: The management dimension of Web-Based Learning covers the maintenance and troubleshooting of the learning environment to ensure proper functioning and distribution of information to the suppliers in the environment. 
vi. Resource Support Dimension: The resource support dimension of the Web-Based Learning Environment covers the online support and resources that are required to ensure hitch-free and meaningful learning environments.

vii. Ethical Dimension: This is a critical dimension of the Web-Based Learning environment, as it relates to several considerations such as socio and cultural diversity, etiquette, legal and regulatory frameworks, geographical diversity, learner diversity, and information accessibility.

viii. Institutional Dimension: The institutional dimension is focused on issues relating to academic affairs and student services from the suppliers (education institutions for example), as it relates to Web-based learning.

\section{THEORETICAL FRAMEWORK}

In this section, we proceed to examine the theoretical frameworks and models that concentrate on the pedagogical aspects of Web-Based Learning. We start with a review of learning theories as applied to Web-Based Learning and thereafter showcase Anderson's (2011) unified theory, which will form the fulcrum of our analysis.

\subsection{Learning Theories for Online Education}

While there is no single theory for online education, some theories have evolved, most of which derive from the major learning theories. In this section, we examine several theories in terms of their relevance and appropriateness for Web-Based Learning.

Community of Online Inquiry (COI). Garrison, Anderson \& Archer (2000) developed the "community of inquiry" model for online learning environments. This model rest on the concept of three different "presences": social, cognitive, and teaching. Anderson, Rourke, Garrison, and Archer (2001) suggested the need for extending the research on each component, as they recognized the overlap and relationship between the three components. Their model provides a framework for the design and delivery of online and blended courses as active communities or learning environments that rely on instructors and students sharing ideas, information, and opinions. Particularly, they noted that "Presence", which is a social phenomenon manifests itself through interactions among students and instructors. The community of inquiry is rapidly becoming a leading model for online and blended courses that are tailored to be highly interactive among learners/students and faculty using blogs, wikis, discussion boards, and videoconferencing.

Connectivism. George Siemens (2004), who is the main proponent of connectivism, presents it as a learning model that recognizes major shifts in the way information and knowledge flows, the pace at which it grows, and way and manner with which it changes because of vast data communications networks. Internet technology has moved to learn from internal, individualistic activities to group, community, and even crowd activities. He describes connectivism as the amalgamation of rules that are discovered by chaos, complexity, network and self-organization theories. Furthermore, this theory sees learning as a process that occurs within nebulous environments of shifting core elements, which is not entirely under the control of the individual. Defining learning as actionable knowledge, Siemens (2004) posits that it can reside outside of ourselves, within a database, or an organization or a database, and it is focused on connecting information sets that are specialized, and the connections and interactions that enable us to learn more (Siemens, 2004). 
Connectivism as a theory thrives on the dynamic of information flow. Students need to understand, and be provided with, experiences in navigating and recognizing rivers of constantly shifting and evolving information. Siemens proposed eight principles of connectivism and presents Connectivism as more suitable for courses/content where enrolment rate is very high, and where the goal or objective of learning is to develop and create knowledge rather than to distribute it.

Online Collaborative Learning (OCL). Linda Harasim (2012) proposed the Online collaborative learning (OCL) theory. The theory focuses on the capabilities of the Internet to create a learning environments that foster collaboration and knowledge building. According to Harasim (2012), OCL focuses on collective learning, knowledge building, and Web use as a means to restructure formal, non-formal, and informal education in a Knowledge Age. Like the Connectivism theory of Siemens, Harasim sees the benefits of moving to teach and learning to the Internet and large scale networked education. In OCL, three key phases of knowledge construction exist through discourse in a group. The first is the generation of the idea. This is the brainstorming phase, where divergent thoughts are gathered. The second phase is the organization of the idea. This is the phase where ideas are contrasted, dissected, and characterized through discussion and argument. The third phase is that of intellectual convergence. This is the phase where intellectual fusion and consensus takes place, including agreeing to disagree, and mostly through a task, essay, or joint piece of work.

OCL benefitted from social constructivism, since learners are encouraged to collaboratively tackle and solve problems through discussions and where the facilitator or teacher plays the role of facilitator as well as learning community member. This is a core area of OCL but also of other constructivist theories, in which the teacher is not necessarily isolated and apart but rather, an involved facilitator of knowledge building. Because of the importance of the role of the teacher, OCL is not easy to scale up. Unlike connectivism, which is more suited for instruction at a largescale, OCL is best positioned in smaller instructional environments. This last issue becomes more prominent when commonality among online education theories is being sought.

Many other theories can be associated with Web-Based Learning, however, we will now turn to present Anderson's (2011) Mode, which acts as a basis for the analysis conducted in this paper.

\subsection{Unified Theory of Web-Based Learning}

Anderson's unified theory is built on three main elements. On the first element, Anderson relied primarily on the well-reputed writings of Bransford, Brown, and Cocking (1999) who were of the opinion that effective learning environments are designed within the union of four critical overlapping lenses: knowledge-centeredness, community-centeredness, assessment centeredness, and learner-centeredness. These four lenses served as the foundational framework that Anderson relied on to build a Web-Based Learning theory, as he examined in detail the characteristics and facilities that the Internet provides with regards to each of the four lenses.

On the second element, Anderson observed that the Internet had progressed from a text-based environment to one that supports different forms of media and are readily available. Furthermore, he noted that the capacity of the Internet's hyperlink is most compatible with the way knowledge is stored and accessed by humans. Finally, and on the third element, Anderson extensively examined the importance of interaction in all forms of learning, and through his evaluation of interaction, he reached a conclusion that interactions are critical components of a theory.

Based on these three elements, Anderson proceeded to construct a model as shown in the figure below. 


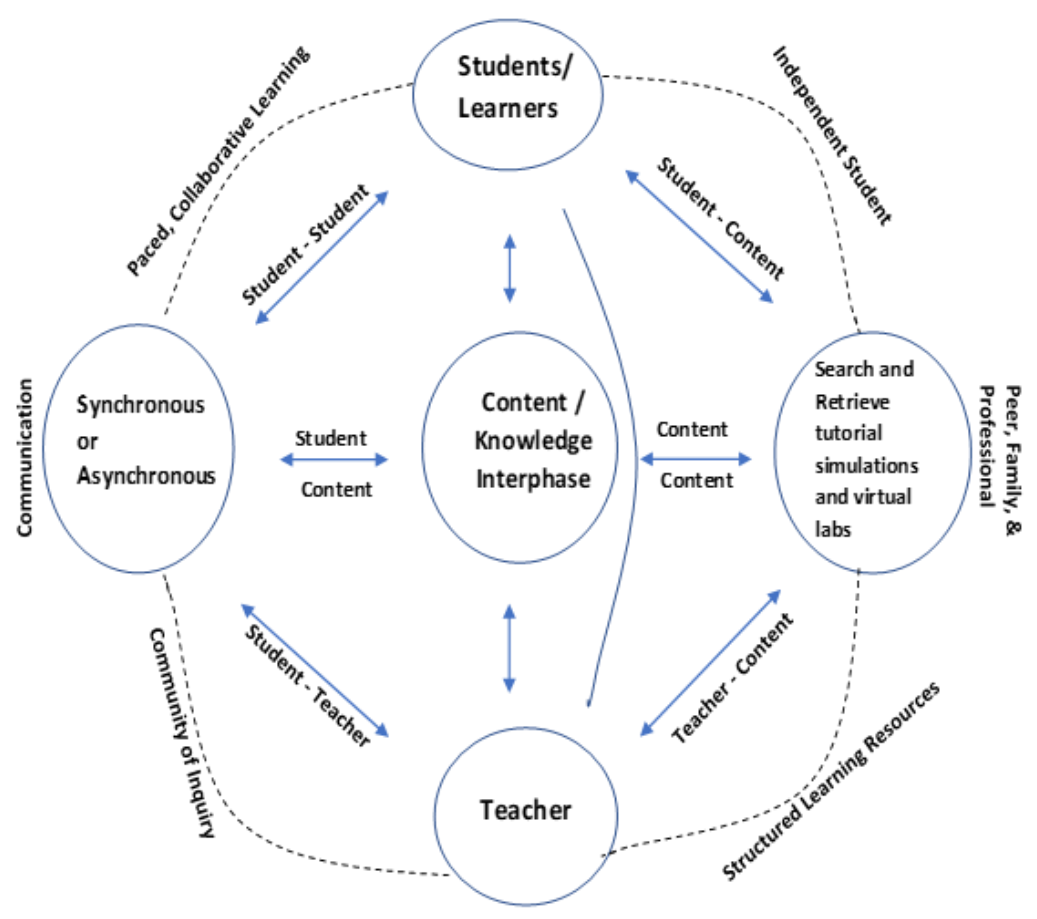

Fig. 2. Anderson's Online Learning Model. (Anderson, 2011)

The two key actors are Students/Learners and Teachers. They also interact amongst each other and with Content. Learners can interact directly with content they find in multiple formats, and especially on the web.

The left-hand side shows interaction that is sequenced, directed, and evaluated with the support of a teacher. This interaction can happen within a community of inquiry using synchronous (realtime) or asynchronous activities (time and place independence). This environment is particularly very rich, primarily because it allows collaborative learning of content, the development of a personal relationship with other learners, and with the teachers. The down-side of this interaction is that it binds participants in time and limits their learning flexibility, as it forces regular sessions or at least a group paced learning.

The right-hand side of the learning shows structured tools associated with independent learning. Essentially, teachers and content providers upload their content and information online, while students and learners search for relevant content and take the courses independently. It shows much less engagement/interaction between different learners, and between learners and teachers. In this mode, the common tools used include computer-assisted tutorials, drills, and simulations (Anderson, 2011).

\section{THEORIZED IMPACT OF COVID-19 ON WEB-BASED LEARNING}

Based on Anderson's (2011) theoretical framework, we will proceed to examine and hypothesized impact of COVID-19 on the two key actors (Learners and Teachers) and interface (Content) of Web-Based Learning, and a further look at this impact across Khan's (2001) eight dimensions of Web-Based Learning as set out in the conceptual framework. 


\subsection{Overall Impact on Learners, Teachers, and Content}

Generally, Student/Learners' interaction will shift more from the left side of the model (StudentStudent interaction and Student-Teacher Interaction) to the right side of the model (StudentContent Interaction). This is primarily because collaborative learning is fundamentally difficult to deliver where students come with different learning needs, pace, background, and access to quality IT infrastructure. The pandemic has made countries mandate a temporal closure of schools, and most schools have moved to online delivery of teaching, and students who hitherto were not used or familiar to the online mode of learning are now forced to go online. Based on Anderson's model, the right-side (self-paced learning with the support of family and professionals) will appeal more to these new entrants into the Web-Based Learning space.

Teachers will be pressured to rapidly fit Content to the Web-Learning environment in the short run. Their interaction with students/learners will increase over the medium to long-run, after fully putting up content. They will thus be also more active on the right-hand side of the model in the short-run and gradually move to the right-hand side in the medium to long run - where active engagement with learners can now begin to take place.

While core Content will largely remain the same, it will, however, require more narratives and explanations with explicit examples, given the fact that Student-Content Interaction will surge more than Student-Teacher interaction and Student-Student interaction. Digitally protection of Content will also surge, as the quality of Content will be a key differentiation. This is critical, as Content explosion due to the surge in Web-Based Learning might lead to poor quality in some instances.

\subsection{Overall Impact across the eight (8) Dimensions of Web-Based Learning}

Expected Impact on Pedagogy Dimension of Student learning. The pedagogy dimension of student learning will be directly impacted by the pandemic, as the number of learners and their requirements increase. While the aims and objectives of learning will not shift significantly, the approach to design, organization, and delivery strategies and methods - by Teachers and institutions shifting Content online- will need to adjust (to accommodate a wider pool of students and learners; this will be more critical in developing countries, where the adoption of Web-Based Learning had been mostly by students of upper-class families (Moeketsi, Matsephe \& Victor, 2018).

Expected Impact on Technological Dimension. Technology-wise, while a range of technological platforms is propping up for learners and teachers alike to utilize, there is an increasing threat to the security of these platforms, especially as regards data and privacy issues. It is also expected, that cost to teachers and learning service providers (such as schools) will rise due to the need to ramp up their technology hardware and software, and to ensure they are well maintained and keep functioning. Learners too will be impacted by the cost of subscriptions to the online learning platforms, as well as to Internet Service providers. For both the developed and developing countries, the efficiency of internet connectivity and services for learners will plummet, due to the surge in traffic on web usage for work and social engagements, as well as the limited ability of ISPs to service their base stations.

Impact on Interface Dimension. Given the rush to Web-Based Learning by a significant number of institutions, learners will experience (at least in the short run), low-quality designs of learning platforms, navigation, and usability testing. In the long run, there will be an overall improvement in the design of learning platforms, as these platforms will adapt, learn, take feedback, and adjust and update. The only aspect of Content that will be impacted by this 
dimension is the overall presentation, which might challenge learners initially, but this is expected to only hold in the short run.

Evaluation Dimension. Evaluation of learners will increasing become challenging for Teachers, especially evaluation of learners that utilize the self-paced and independent learning. This will be more critical in the short-run, where there is an increasing influx of learners. Teachers will need to adjust and implement a more robust evaluation process. Ultimately, learners will, therefore, be exposed to a more rigorous evaluation process, to ensure the sanctity and reliability of outcomes from Web-Based Learning. In terms of the overall instruction and learning environment, some platforms will compromise to attract increased utilization by learners, this will lead to certification of platforms, and implementation of certain controls such as subscriptions by platforms and learners to certain Mutual Accountability Framework.

Impact on Management Dimension. There will be an increasing requirement for the management of platforms as a result of the surge in Web-Based Learning. Institutions moving to Web-Based teaching, or already utilizing it, will need to scale up their resources (human and technical) to ensure quick maintenance of its platforms to ensure minimal downtime. A system for automatic troubleshooting and reporting procedure will need to be instituted. Teachers and learners will more often than before experience little glitches in the utilisation of IT platforms, they thus need to learn to properly describe and promptly report challenges in the utilization of platforms. Management of Content will be key too, as it might be affected sometimes, and thus owners of Content will need to implement a robust data storage/backup system for their Content.

Resource Support Dimension. Given the fact that some teachers and learners who are not too conversant with the workings of the internet, and had hitherto stayed away, are now being compelled to utilize it, there will be increasing need of resource support to ensure easy utilization of online learning platforms, to ensure meaningful learning environments. New tools for migrating Content (from a hard copy) to the Web-Based Learning format will also need to be created, to allow for ease, speed, and efficiency.

Impact on Ethical Dimension. According to Van Reijswoud (2009), appropriate internet technologies are those which are "suitable for the cultural, environmental, organizational, economic and political conditions in which it is intended to be used" Ethical considerations and related issues will rise to the front burner. This will be aggravated by cultural and religious diversity (as Web-Based Learning creates a limited opportunity for Cultural and Religious customization of learning medium and delivery). This is expected to trigger legal and regulatory issues, which will later pose as a challenge to some learning platforms, and make delivery more complex and expensive. This dimension will directly affect the Teacher and the Content (supply side), and indirectly affect the learners (demand side).

Impact on Institutional Dimension. In terms of institutional dimension, academic affairs will require a new set of skills, and the bottom line will be impacted in the short-run, as they cannot fully transfer the cost of increased utilization of Web-Based learning Platforms for instructional delivery. The pricing model will change, but its ultimate impact and direction price remain ambiguous. There will however be increased competition for academic institutions.

Learners will have a broader pool of institutions to take classes from, both locally and internationally, and so geo-location will no longer be a competitive offering of academic institutions. Teachers will increasingly be able to moonlight (sell their services and skill to different competitors). While restrictions can be put in place to manage this, issues of plagiarism, copyright, and patent will surge, calling for increased regulations. This will be a prominent issue 
for developing countries, which have poorly developed institutional, legal, and regulatory frameworks for web/online related activities (Moeketsi, Matsephe \& Victor, 2018).

\subsection{Impact of COVID-19 on Access to Quality Learning}

"Creative and innovative applications of information and communication technologies (ICTs) have long been seen as important potential tools to enable educational reform processes improving both access to education, and the quality of that education" (The World Bank, 2010). While the internet and technological tools can increase access to education through distance learning, enabling a knowledge network for students, support teacher training, and broaden the availability of quality education materials, it is pertinent to note that implementation requires significant time, resources, and efforts to make it a worthwhile and useful exercise. COVID-19 was a sudden shock to Teachers and Learners, even the most prepared was hit by the sudden need to adjust with little or no time to prepare a phased migration or increase capacity for WebBased Teaching and Learning. Based on this shock and as expressed by the analysis of the eight dimensions of learning, it is expected that access to quality learning through the web will be poor, especially when juxtaposed with face to face classroom interaction. This will especially be true for developing countries, where there are strong inequalities when it comes to access to technology and digital devices (see Counted and Arawole, 2016; Alison, 2017). Many learners in these countries suffer a form of digital inequality whereby they lack the connections, devices, and skills to learn remotely.

\section{Challenges to Web-Based Learning Environment in Periods of CRISIS, AND EFFICIENT APPROACHES AND TECHNOLOGIES REQUIRED FOR IMPROVEMENTS}

We categorize the key challenges and required changes to the web-based learning environment from three perspectives; the supply side (providers of the learning platform and assess to the platform, as well as the content creators/developers) and the demand side (the users of the web based learning environment) and the institutions side (the institutions that moderate between the supply and the demand side, and ensures the efficient and effective functioning of the web based learning environment).

From the supply (provider) side, the key challenges and their related improvements are;

i. Cost of Technology: the chain of activities required to transition to a web-based learning environment (which starts from the planning stage to the design stage, and then the implementation and adoption stage), all require finance, which in some instances might be expensive for most institution to cover, given their unpreparedness to such a sudden and swift shift that COVID-19 has necessitated.

Solution: To navigate this challenge, learning institutions can leverage existing web-based learning platforms, instead of fully designing and adopting their own individual platform from the onset. Leveraging existing platforms is faster and more cost effective. However, given the level of dependency and risk associated with not owning a web-based learning platform, learning institutions should have a medium to long term plan that will allow transition to their own platform. Another consideration should be collaborating with similar institutions in rolling out a platform. Here, cost is shared amongst collaborating learning institutions.

ii. Insufficient time to design a robust web based learning course: Given the shock of the COVID-19pandemic, and the rush to move learning online by most institutions, insufficient 
time spent on course development and design would most likely arise, and it will be a big contributor to poorly development learning experience and a critical challenge for both elearning instructors and for learners. According to Archambault (2010), the amount of time required to design, develop, and roll out a well-designed web-based learning programmeis a critical consideration, and this is so because of new content, emerging technologies, and new methods of engaging online learners.

Solution: one of the ways content developers can overcome this challenge is by collaborations with other developers within their e-learning professional communities. This collaboration and discourse can help reduce the overall time spent in designing and implementing a robust online content.

iii. Platform Security: the increasing utilization of web-based learning platform has created a new target for criminals, who hack into servers, or hijack platforms for selfish gains. This challenge is real, especially as the web-based learning platform mostly has a lot of information ranging from courseware, school information, student's information etc. For effective security, measures should include ensuring learner/users are duly registered by the system and institute measures to prevent Cross-site scripting (XSS) which is a means for third-party to get access to a web system. Other measures should include use of alphanumeric character (Upper and lower) for Passwords for admin and learners, and in some case, inclusion of signs and symbols that adds additional layers of protection. To also prevent a total crack down of the system at any given time, individual systems within the overall system should be given a unique password which should be different from others, and where possible, different machines/servers should be used for different services. Frequent system update to understand how the learning platform is been used is also critical. Recovery procedure should be instituted within the system and all information should be frequently backed up. Where expertise within the institution of learning is lacking, IT security expert should be engaged in managing the system.

iv. IT Capability of platform managers: When personnel handling the web-based platform (including the instructional aspect) of institutions are inexperienced, it could create more trouble for both the institution and the users. It is very important that competent managers are engaged to manage web-based learning platforms

v. Infrastructure availability: for developing countries like Nigeria, Power and Internet are key issues, and their availability are critical requirements to successfully adopt a web-based learning platform. This creates an added layer of cost for the institutions that are rolling out web-based learning platforms. Managing these challenges requires collaborations to drive down cost.

From the demand (user) side, the key issues are;

vi. Digital Divide: the digital divide is the primary challenge for most learners in the webbased learning space. It is commonly defined as the gap between learners who have access to computers and connectivity, and those who do not have, as well as those who have the knowledge on how to use the internet and other information technologies, and those who do not have this knowledge (Bernard, 2011; Hall, 2013). It is pertinent to note that due to the declining cost of many information technologies today, the meaning of digital divide is shifting from having access, to knowing how to use the technologies (Bernard, 2011). While this is true in developed countries, same cannot be said of emerging economies like Nigeria, where income levels are still low, and a vast majority of the populace still live in poverty. The digital divide therefore still acts as a challenge for education, and more specifically web-based learning environments. 
Solution: one way to address this challenge is for the instructor to implement a learning environment that fosters collaboration. Again, providing learners/users with the opportunity to collaborate, share, and create will improve theuser's use of diverse technologies, enhance their web-based learning experience, and support self-directed and ongoing learning. It is critical, that instructors access the user/learners technological competence and access, and offer the learners the opportunity to grow, while providing added support. Allowing offline functionality of platforms as well as ability to run on multiple technological platforms will also help in reducing the digital divide.

vii. Learners Adaptability. A rapid switch from a traditional classroom face to face learning to an online environment makes the learning experience entirely different for learners. For instance, while passive listening and note taking are expected in a traditional classroom, online discussions fostered by web-based learning demands moving to action immediately. It is often difficult for students with the "traditional" mindset to adapt. Solution: making learners understanding the benefits of web-based learning and even engaging them in discussions with their peers may change this mindset and position them to better utilize the online classes. Online forums should be maximized. Webinars, group work or forums where learners can discuss and resolve their questions are critical. It is also very important, that students have a teacher they can contact, for instance, tutoring via Skype.

viii. Technical Issues: technical challenges are one of the main challenge students face in webbased learning. Compatibility issues (with operating system, web browsers or phone), low bandwidth or low internet connection, adds to learning frustration and student-instructor engagement. as properly equipping themselves for the course's successful completion.

Solution: developers of web-based learning platforms will need to provide the opportunity for multi-device courses and personal attention. Also web-based learning courses should be designed in a way and manner that do not require huge internal memory or a high-speed Internet connection, and with a solid and simple script. Web-based learning platforms should have integrated into them, a simple and comprehensive help page, a robust Frequently Asked Question Section, and a good student care service. there should also be room for chat service, email address or forum for addressing technical glitches

ix. Inaction from flexibility: the flexibility that comes with web-based learning has the potential to trigger inaction from learners. Most times, students can take courses when and where they like, at their own pace and with no physical limitations and this may result in student not accessing the learning platform or completing the course. There is the psychology element to this, as they assume they have so much time and flexibility and may never actually find time to do it.

Solution: addressing this requires setting time limit and sending reminders. Setting up clear and simple calendar indicating when learners should have completed each part of the online course is critical. In addition, setting reminders to students telling them that they are running out of time and encouraging them to complete the course. It will also require dividing courses into multiple parts and short lessons that can be completed in a short amount of time.

From the regulatory standpoint (the government), the key issue is that of protection of Intellectual Property of owners of web-based learning platforms, to ensure that innovation is encouraged, and that unguarded market disrupting behaviour and excesses that foster undue profiting and advantages to certain platforms over others do not arise. Users should also be protected from exploitative tendencies of service providers. It will also be critical, that the government provides robust internet security architecture to help secure increasing activities on the web. Necessary policies, and laws that will foster these needs to be instituted. 


\section{SuMMARY AND CONCLUSION}

Learners are generally responding to quarantine and large-scale shutdown (partial) of cities with a sudden shift to Web-Based Learning. However, given that a pandemic of this nature and scale has never been experienced in recent history, there is a knowledge gap as to how teachers and learners should respond to the shift, and what the likely impact and the key considerations should be. This paper is an early attempt at hypothesizing the impact of the pandemic on two key actors (Learners and Teachers) and one interface (Content), all across eight dimensions. The analysis is based on Khan's (2001) dimension of Web-Based Learning and Anderson's (2011) theory on Online Learning. Building this knowledge base is critical, as this paper shapes the thinking of stakeholders as it sets out the factors that will drive the effectiveness of learning when taken from the classroom and transposed to technological devices.

Overall, the pandemic has delivered severe shocks to both the demand and supply side of WebBased Learning, with Leaners, Teachers, and Content all significantly affected. While we hypothesize a general drop in the quality of teaching and learning in the short run, we expect the opposite to be the case in the long run, when the demand and supply side self-correct (guided anyway by strong government and market institutions).

It is pertinent to note that the above hypothesis is based on an analysis of the dimensions of WebBased Learning and careful observation of actors and interface. For future research, when Post COVID-19 Web-Based Learning data becomes more readily available, these hypotheses can be subjected to more empirical rigor and data analysis to validate the direction and magnitude of the impact of COVID-19 on Web-Based Learning

\section{REFERENCES}

1. Alison, G. (2017). Beyond Access: Addressing Digital Inequality in Africa. Centre for International Governance Innovation and Chatham House. Paper Series: No. 48

2. Anderson, T. (2011). The theory and practice of online learning (2nd Edition). Edmonton, AB: AU Press.

3. Anderson, T., Rourke, L., Garrison, D.R, and Archer, W. (2001). Assessing social presence in asynchronous text-based computer conferencing. Journal of Asynchronous Learning Networks, 5(2) Retrieved from: http://immagic.com/eLibrary/ARCHIVES/GENERAL/ATHAB_CA/Anderson.pdf

4. Archambault, L. (2010). Identifying and addressing teaching challenges in k-12 online environments. Distance Learning, 7(2), pp. 13-17.

5. Counted, A. and Arawole, J. (2016). We are connected, but constrained': internet inequality and the challenges of millennials in Africa as actors in innovation. Journal in Innovation and Entrepreneurship 5(3), https://doi.org/10.1186/s13731-015-0029-1

6. Dalton, K. and Gren, I. (2015). Barriers and strategies on adoption of e-learning in Tanzanian higher learning institutions: Lessons for adopters. International Journal of Education and Development using Information and Communication Technology (IJEDICT);11(2),126-137.

7. Garrison, D. R., Anderson, T., and Archer, W. (2000). Critical inquiry in a text-based environment: Computer conferencing in higher education model. The Internet and Higher Education, 2(2-3), 87105.

8. Gennaioli, la Porta, Lopez-de-silanes and Shleifer, (2011). Human Capital and Regional Development, NBER working Paper 17158.

9. Ghavifekr, S. and Rosdy, W.A.W. (2015). Teaching and learning with technology: Effectiveness of ICT integration in schools. International Journal of Research in Education and Science (IJRES), 1(2), 175-191.

10. Harasim, L. (2012). Learning theory and online technologies. New York: Routledge/Taylor \& Francis.

11. Kahn, B. H. (2001). Web-based training: An introduction. In B.H. Kahn (Ed.) Web-based Training. Englewood Cliffs, NJ.: Educational Technology Publications. 
International Journal of Computer Science \& Information Technology (IJCSIT) Vol 12, No 3, June 2020

12. Kwanjai D. (2014). The design of web-based learning model using collaborative learning techniques and a scaffolding system to enhance learners' competency in higher education. 5th World Conference on Educational Sciences - WCES 2013. Procedia - Social and Behavioral Sciences; 116, 436 - 441

13. Moeketsi Letseka, Matsephe Martha Letseka and Victor Pitsoe (November 5th 2018). The Challenges of E-learning in South Africa, Trends in E-learning, Mahmut Sinecen, IntechOpen, DOI: 10.5772/intechopen.74843. Available from: https://www.intechopen.com/books/trends-in-elearning/the-challenges-of-e-learning-in-south-africa

14. Nagunwa, T. and Lwoga, E. 2012. "Developing eLearning technologies to implement competency based medical education: Experiences from Muhimbili University of Health and Allied Sciences", International Journal of Education and Development using Information and Communication Technology (IJEDICT), vol. 8, no. 3, pp. 07-21.

15. Picciano, A. G. (2017). Theories and frameworks for online education: Seeking an integrated model. Online Learning, 21(3), 166-190. doi: 10.24059/olj.v21i3.1225

16. Said H. (2010). A Conceptual Framework for Using and Evaluating Web-Based Learning Resources in School Education. Journal of Information Technology Education; 9,53-79.

17. Sanga, C., Magesa, M. M., Chingonikaya, E. and Kayunze, K. A. 2013. "Can e-learning promote participation of female students in STEM disciplines in higher learning institutions of Tanzania?", International Journal of Education and Development using Information and Communication Technology (IJEDICT), vol.9, no.3, pp. 86-102.

18. Siemens, G. (2004). Connectivism: A learning theory for the digital age. Paper retrieved from: http://www.elearnspace.org/Articles/connectivism.htm

19. Sudha, A. and Amutha S. (2015) Higher Secondary Learners' Effectiveness towards Web Based Instruction (WBI) on Chemistry. Universal Journal of Educational Research, 3(7): 463-466. DOI:10.13189/ujer.2015.030706

20. The European-wide e-Learning Recognition Review Report (2015). Retrieved from: http://www.elearningnews.it/_resources/images/ebook/european-wide-elearningrecognitionreview.Pdf.

21. The World Bank. (2010). ICTs \& education: Issues and opportunities. Report. Retrieved June 30, 2011 from http://siteresources.worldbank.org/EDUCATION/Resources/ESSU/4632921290618190826/Background_Note_EducationStrategy2020_ICT_Edu.pdf

22. Titiana C (2019). Design of Web-Based Learning Model: Shifting the Accent from Knowledge Transmission to Knowledge Construction. Proceedings of the Central and Eastern European, 177-189

23. Todaro, M. and Smith, S. (2011). Economic Development, (11th ed.), Addison Wesley, 2011.

24. Van Reijswoud, V. (2009). Appropriate ICT as a tool to increase effectiveness in ICT4D: Theoretical considerations and illustrating cases. The Electronic Journal on Information Systems in Developing Countries, 38(9), 1-18.

25. Yongxia, D. and Peili Z. (2018). Practice and effectiveness of web-based problem-based learning approach in a large class-size system: A comparative study. Nurse Education in Practice, 31;161-164 